PROCEEDINGS OF THE

AMERICAN MATHEMATICAL SOCIETY

Volume 132, Number 3, Pages 697-698

S 0002-9939(03)07180-6

Article electronically published on September 29, 2003

\title{
A SIMPLE PROOF THAT SUPER-REFLEXIVE SPACES ARE $K$-SPACES
}

\author{
FÉLIX CABELLO SÁNCHEZ
}

(Communicated by Jonathan M. Borwein)

AbstraCt. We demonstrate the title.

A quasi-Banach space $Z$ is called a $K$-space $[3$ if every extension of $Z$ by the ground field splits; that is, whenever $X$ is a quasi-Banach space having a line $L$ such that $X / L$ is isomorphic to $Z, L$ is complemented in $X$ (and so, $X=L \oplus Z$ ). These spaces play an important rôle in the theory of extensions of (quasi) Banach spaces [1], [2].

The property of being a $K$-space is closely related to the behaviour of quasilinear functionals. Recall that a homogeneous functional $f: Z \rightarrow \mathbb{K}$ is said to be quasi-linear if there is a constant $Q$ such that

$$
|f(x+y)-f(x)-f(y)| \leq Q(\|x\|+\|y\|) \quad(x, y \in Z) .
$$

The least possible constant in the preceding inequality shall be denoted by $Q(f)$.

It is well known [1] that $Z$ is a $K$-space if and only if each quasi-linear functional on $Z$ can be approximated by a true linear (but not necessarily continuous!) functional $\ell: Z \rightarrow \mathbb{K}$ in the sense that the distance

$$
\operatorname{dist}(f, \ell) \stackrel{\text { def }}{=} \inf \{K \geq 0:|f(x)-\ell(x)| \leq K\|x\| \text { for all } x \in Z\}
$$

is finite.

The main examples of $K$-spaces are supplied by Kalton and co-workers: for instance, $\mathcal{L}_{p}$ spaces $(0<p \leq \infty)$ are $K$-spaces if and only if $p \neq 1$ ([1], [4], [5], [6]). Also, $B$-convex spaces (Banach spaces having nontrivial type $p>1$ ) are $K$-spaces and so are quotients of Banach $K$-spaces.

In this short note, we present a very simple proof that super-reflexive Banach spaces are $K$-spaces. Of course this is contained in Kalton's result for $B$-convexity. Nevertheless, I believe that a simpler proof for this particular case is interesting because, in the presence of some unconditional structure (e.g., for Banach lattices), $B$-convexity is equivalent to super-reflexivity.

Mini-Theorem. Every super-reflexive space is a $K$-space.

Proof. Suppose on the contrary that $Z$ is super-reflexive and there exists a quasilinear function $f: Z \rightarrow \mathbb{K}$ such that $\operatorname{dist}(f, \ell)=\infty$ for all linear maps $\ell: Z \rightarrow \mathbb{K}$.

Received by the editors June 20, 2001.

2000 Mathematics Subject Classification. Primary 46B03, 46B08, 39B82.

Key words and phrases. $K$-space, super-reflexivity, ultraproduct.

Supported in part by DGICYT project BMF 2001-083.

(C)2003 American Mathematical Society 
Let $\mathcal{F}$ denote the family of all finite-dimensional subspaces of $Z$. For each $E \in \mathcal{F}$, let $f_{E}$ denote the restriction of $f$ to $E$. It is clear that $Q\left(f_{E}\right) \leq Q(f)$. Put

$$
\delta_{E}=\operatorname{dist}\left(f_{E}, E^{*}\right)=\inf \left\{\operatorname{dist}\left(f_{E}, \ell\right): \ell \in E^{*}\right\} .
$$

Obviously, $\delta_{E}$ is finite for all $E \in \mathcal{F}$. The hypothesis means that $\delta_{E} \rightarrow \infty$ with respect to the natural (inclusion) order in $\mathcal{F}$. In particular, $\delta_{E}>0$ for $E$ large enough. Now, for each $E \in \mathcal{F}$, take $\ell_{E} \in E^{*}$ such that $\operatorname{dist}\left(f_{E}, \ell_{E}\right)=\delta_{E}$ and let $g_{E}=\delta_{E}^{-1}\left(f_{E}-l_{E}\right)$ (if $\delta_{E}=0$, take $\left.g_{E}=0\right)$. Clearly, $\left|g_{E}(x)\right| \leq\|x\|$ provided $x \in E$. Also, it is clear that $Q\left(g_{E}\right) \rightarrow 0$ as $E$ increases in $\mathcal{F}$.

Let $\mathfrak{V}$ be any ultrafilter refining the Fréchet filter on $\mathcal{F}$, and let $\mathcal{F}_{\mathfrak{V}}$ denote the ultraproduct of $\mathcal{F}$ with respect to $\mathfrak{V}$. Define $g: \mathcal{F}_{\mathfrak{V}} \rightarrow \mathbb{K}$ by

$$
g\left[\left(x_{E}\right)\right]_{\mathfrak{V}}=\lim _{\mathfrak{V}(E)} g_{E}\left(x_{E}\right),
$$

where $\left[\left(x_{E}\right)\right]_{\mathfrak{V}}$ denotes the class of $\left(x_{E}\right)$ in $\mathcal{F}_{\mathfrak{V}}$.

Obviously, $g$ is a (well-defined) bounded linear functional on $\mathcal{F}_{\mathfrak{V}}$ and, in fact, $\|g\| \leq 1$. Since $Z$ is super-reflexive, $\mathcal{F}_{\mathfrak{V}}$ is reflexive and we have $\left(\mathcal{F}_{\mathfrak{V}}\right)^{*}=\left(\mathcal{F}^{*}\right)_{\mathfrak{V}}$, where $\mathcal{F}^{*}=\left\{E^{*}: E \in \mathcal{F}\right\}$ (see 7$]$ ). It follows that $g=\left[\left(\ell_{E}^{\prime}\right)\right]_{\mathfrak{V}}$, where $\ell_{E}^{\prime} \in E^{*}$ and $\left\|\ell_{E}^{\prime}\right\| \leq 1$ for all $E$. Hence,

$$
\lim _{\mathfrak{V}(E)} g_{E}\left(x_{E}\right)=\lim _{\mathfrak{V}(E)} \ell_{E}^{\prime}\left(x_{E}\right)
$$

and so

$$
\lim _{\mathfrak{V}(E)} \operatorname{dist}\left(g_{E}, \ell_{E}^{\prime}\right)=0 .
$$

In particular, for every $\varepsilon>0$, the set $\mathcal{S}=\left\{E \in \mathcal{F}: 0<\operatorname{dist}\left(g_{E}, \ell_{E}^{\prime}\right)<\varepsilon\right\}$ belongs to $\mathfrak{V}$. But, for $E \in \mathcal{S}$, one has

$$
\operatorname{dist}\left(f_{E}, \ell_{E}+\delta_{E} \ell_{E}^{\prime}\right) \leq \varepsilon \delta_{E}<\delta_{E},
$$

a contradiction.

\section{REFERENCES}

[1] N. Kalton, The three space problem for locally bounded $F$-spaces, Compositio Mathematica 37 (1978) 243-276. MR 80j:46005

[2] N. Kalton, Nonlinear commutators in interpolation theory, Memoirs of the American Mathematical Society, vol. 73, no. 385, 1988. MR 89h:47104

[3] N. Kalton, N. T. Peck, and J. W. Roberts, An F-space sampler, London Mathematical Society Lecture Note Series 89, Cambridge University Press, Cambridge, 1984. MR 87c:46002

[4] N. Kalton and J. W. Roberts, Uniformly exhaustive submeasures and nearly additive set functions, Trans. Amer. Math. Soc. 278 (1983) 803-816. MR 85f:28006

[5] M. Ribe, Examples for the nonlocally convex three space problem. Proc. Amer. Math. Soc. 73 (1979) 351-355. MR 81a:46010

[6] J. W. Roberts, A nonlocally convex $F$-space with the Hahn-Banach approximation property, in: Banach spaces of analytic functions, Springer Lecture Notes in Mathematics 604, BerlinHeidelberg-New York (1977) 76-81. MR 58:30008

[7] B. Sims, "Ultra"-techniques in Banach space theory. Queen's Papers in Pure and Applied Mathematics 60, Queen's University, Kingston, Ontario, Canada, 1982. MR 86h:46032

Departamento de Matemáticas, Universidad de Extremadura, Avenida de Elvas, 06071 BADAJOZ, SPAIN

E-mail address: fcabello@unex.es 